\title{
Abstract of the mean blood pressure in preterm infants
}

\begin{abstract}
Objective: The hypothesis of this study was that low mean blood pressure (LMBP) in stable extremely low birth weight (ELBW) infants does not require treatment with either volume overload or vasopressors and left to right shunting via patent ductus arteriosus (PDA) in these infants may be attributed to LMBP.

Study design: Thirty-four ventilator dependent infants born at 24 to 28 week gestation, clinically stable with MBP less than the 10th percentile for their gestational age during the first 24 hours of life, were prospectively randomized into two groups. The treatment group received either intravenous fluid boluses or vasopressors (dopamine, dobutamine) determined by the neonatologist, and the control group received no treatment. Systolic, diastolic, and MBP were obtained by umbilical arterial catheter (UAC) during the study period. Diagnosis of PDA was confirmed with the use of twodimensional echocardiographic imaging and Doppler color flow mapping.
\end{abstract}

Results: Serial blood pressure measurements in the treated group were comparable to the control group. There were no significant differences between the initial MBP during the first 24 hours of life ( $23.7 \pm 2.7$. vs. $24 \pm 3$ torr), systolic ( $34.4 \pm 5.3$ vs $35.2 \pm 3.4)$ and diastolic blood pressure, $(17.2 \pm 2.9$ vs. $17.9 \pm 27$ torr $)$ between the treatment and the control groups respectively. All infants (17) in the treatment group were given fluid boluses, four infants also received dopamine, and two of these 4 infants were also administered dobutamine. There were significant differences in systolic, diastolic and mean blood pressure, before and after PDA treatment within each group on 4th and 7 th days respectively $(\mathrm{P}<0.01)$ but not between the two groups. Six infants in the study group did not have PDA and their blood pressure continued to increase during the study period. Intraventricular hemorrhage was significantly higher in the treatment versus the control group respectively $(\mathrm{P}<0.03)$.

Conclusion: The study suggests that low mean blood pressure in stable ELBW infants does not require treatment with either fluid boluses or vasopressors. PDA with left to right shunt may have been responsible for low MBP in $80 \%$ of this study population. Treatment and closure of PDA increased mean blood pressure which continued to increase during the first week of life. The use of Intravenous fluid boluses and vasopressors may potentially increase the risk for IVH.

Keywords: ELBW, IVH, PDA, ABV, ECHO
Volume 4 Issue 2 - 2018

\author{
Ibrahim H,' Kasli T, ${ }^{2}$ Tice H,' Caldito G,' \\ Sangster G,' Huda S, ${ }^{3}$ Pramanik A' \\ 'Department of Pediatrics, LSU Health-Shreveport, USA \\ ${ }^{2}$ Children's Health \& Diseases, Kocaeli, Turkey \\ ${ }^{3}$ Department of Pediatrics, Christus St. Frances Cabrini Hospital, \\ Egypt
}

Correspondence: Ibrahim H, Department of Pediatrics, LSU Health-Shreveport, LA, USA, Tel +318 626-1622, Fax 318 6984305, Email HIbrah@Isuhsc.edu

Received: February 15, 2018| Published: April 18, 2018

\section{Introduction}

Significant proportion of premature infants admitted to neonatal intensive care units receive treatment for hypotension, there is lack of based therapeutic intervention to assess its effectiveness. ${ }^{1,2}$ Several authors suggested to treat hypotension in the newborn is based on statistically defined gestational and postnatal age-dependent normative blood pressure values; approximately the 10th percentile for gestational age. ${ }^{3,4}$ The Joint Working Group of the British Association of Perinatal Medicine has recommended that the MBP in $\mathrm{mmHg}$ should be maintained at or greater than the gestational age in weeks. ${ }^{5}$ The rational for therapeutic support of blood pressure in ELBW infants is often based on these assumptions.

The immature cardiovascular system and its dynamic physiology in these low birth weight (ELBW) infants contribute to a wide range of observed arterial blood pressure values $(\mathrm{ABV})$ in the first week of life. In premature infants factors influence hemodynamic stability include myocardial function, patent ductus arteriosus (PDA) shunt, labile pulmonary and systemic vascular resistance, and the use of mechanical ventilation. ${ }^{6,7}$ Studies are fraught with defining normal ranges of $\mathrm{ABV}$ in the premature infants design limitations. ${ }^{8-11}$ Due to lack of autoregulation of cerebral perfusion in premature infants, sudden increase in blood pressure may have adverse consequences such as cerebral and other organ injury. ${ }^{12}$ There are differences among organs in the critical point of oxygen delivery: gut oxygen delivery decreases earlier in hypotension than oxygen delivery to other organs. The consequence of inadequate perfusion is ischemic injury to the kidney, gut, brain, and myocardium followed by multiple organ failure.

The primary objective of this study to evaluate whether treating low mean blood pressure (LMBP) in stable ELBW infants in the first 24 hours of life has advantage over no treatment. The secondary objective was to determine the role of the PDA with left to right shunt in decreasing MBP.

\section{Methods}

This prospective, randomized clinical study was performed at a 
single, regional referral level III neonatal intensive care unit. The study protocol was approved by the human research review board. Written informed consent was obtained antenatally from all mothers in premature labor.

\section{Patient population}

Eligible infants were enrolled between January 2007 and December 2009. Inclusion criteria were clinically stable inborn premature infants with a gestational age less than or equal to 28 weeks, and birth weight equal to or less than 1000 grams, with MBPs less than the 10th percentile for their gestational age that continued to be lower than the target mean blood pressure (MBP) for 4 hours in the first 24 hours of life. Gestational age was determined by first trimester ultrasound or by obstetrical dating of the pregnancy and examination of the newborn. All infants were mechanically ventilated, and were clinically stable with good capillary perfusion less than 3 seconds, urinary output of greater than $1 \mathrm{ml} / \mathrm{kg}$ /hour, base excess \pm 4 , and pulse oximeter greater than or equal to 88 percent (Nelcore pulse oximeter used in our unit). Infants with congenital anomalies, early sepsis, maternal chorioamnionitis, and abruption placenta were excluded.

On admission to the neonatal intensive care unit (NICU), systolic, diastolic, and mean blood pressures (ABVs) were measured continuously with a disposable pressure transducer (Edward life science) connected to a single lumen umbilical arterial catheter filled with saline, and positioned between T-6 and T-9 into the abdominal aorta. This system was calibrated with zero reference at mid-chest level. A continuous pressure wave and digital recordings were displayed on a neonatal monitor (GE-2000). An echocardiogram (ECHO) was ordered within $24 \mathrm{~h}$ of study entry. PDA with left to right shunt was defined as: tachycardia at rest (heart rate $>165 \mathrm{bpm}$ ), bounding pulses, wide pulse pressures $(>20 \mathrm{~mm} \mathrm{Hg})$, systolic murmur consistent with a PDA, radiographic evidence of cardiomegaly or increased pulmonary vascular markings, increasing ventilator or oxygen requirements, and/or worsening metabolic acidosis. PDA was confirmed by an ECHO when the evaluation showed a ductal diameter $>1.5 \mathrm{~mm}$ left to right shunt was diagnosed of retrograde diastolic flow was rated in the descending aorta and a left atrium to aortic root ratio of $>1.4$.

\section{Randomization}

A computer generated randomization numbers are utilized to assign infants into treatment versus control groups.

\section{Treatment strategies}

We compared treatment with intravenous normal saline hours of $20 \mathrm{ml} / \mathrm{kg}$ over 30 minutes and/or dopamine/dobutamine to keep the MBP at or above the gestational age in weeks in the treatment group versus observing several blood pressure measurements in the control group.

\section{Primary outcomes}

The primary outcomes were to compare systolic, diastolic and MBP on day one, four and seven of life between the treatment and control groups and within each group before and after treatment for closure of PDA in those with left to right shunt.

\section{Secondary outcomes}

Secondary outcomes included death before discharge home,
Bronchopulmonary dysplasia (BPD) in survivors with oxygen required at 36 weeks of postmenstrual age (PMA), IVH on ultrasonography of the head performed at $\leq 36$ weeks PMA, retinopathy of prematurity (ROP) requiring laser therapy or surgery, and intestinal perforation or necrotizing enter colitis (NEC) according to Bell staging. ${ }^{13,14}$

\section{Statistical analysis}

The chi-square or Fisher test was used to compare the control and the treatment groups on categorical variables, whereas the Wilcoxon rank sum test was used to compare the numerical variables. The two sample t-test (if variable was normally distributed in both groups) or the Wilcoxon rank sum test (if variable was not normally distributed in either group) was used to compare them on numeric factors. The treatment and control groups were compared on baseline characteristics, primary and secondary outcomes. Observed differences between the control and treatment groups were considered significant if comparable $p$ values were less than 0.05 , with a power of $80 \%$ to detect a $20 \%$ difference in the mean blood pressure between the two groups). Repeated Measures ANOVA was performed to determine significant effects of group (Control versus Treatment) (First 3 days, Day 4 and Day 7) with time as the repeated measure. Subject (nested within group), group and time were tested for their significant effects on BP. If time had a significant overall effect, the Bonferroni multiple comparison test was used to determine significant 2-time comparisons of average BP for the three time periods during the week observation of BP measurements. SAS version 9.2 was used for statistical computing.

\section{Results}

One-hundred and sixty-six infants were admitted to our NICU between January 2007 and December 2009, with 90 infants born at 28 weeks or less gestation. Thirty-four infants were eligible for the study. All infants were less than 1000grams with gestational ages between 24 and 28 weeks. The treatment and control groups were compared on baseline characteristics, primary, and secondary outcomes which were comparable (Table 1).

Table I Baseline demographic Characteristics between the two groups

\begin{tabular}{llll}
\hline & Treatment & Control & \\
& $(\mathbf{N}=17)$ & $\mathbf{( N = 1 7 )}$ & \\
\hline Male sex - no. $(\%)$ & $9(52.9)$ & $8(47.1 .0)$ & 0.73 \\
Gestational age-week & $25.8 \pm 1.5$ & $25.9 \pm 1.7$ & 0.73 \\
Birth weight & $692 \pm 157$ & $8 \mathrm{II} \pm 16 \mathrm{I}$ & 0.08 \\
Apgar at I minute & $5(2-7)$ & $5(3-8)$ & $0.6 \mathrm{I}$ \\
Apgar at 5 minute & $7(4-8)$ & $7(3-8)$ & 1 \\
Antenatal steroids & $7(44 \%)$ & $\mathrm{II}(64 \%)$ & 0.23 \\
Pre-eclampsia & $3(17.6 \%)$ & $4(23.5 \%)$ & $>0.99$ \\
Chorioamnionitis & $3(17.6 \%)$ & $2(1 \mathrm{I} .8 \%)$ & $>0.99$ \\
Intra-partum & $3(17.6 \%)$ & $3(17.6 \%)$ & $\mathrm{I}$ \\
hemorrhage & & & \\
Cesarean section & $13(76.5 \%)$ & $12(70.6 \%)$ & 0.99 \\
\hline
\end{tabular}

\section{Primary outcome}

The systolic, diastolic and MBP values were decreased in the first 
24hours of life, and continued to be below the 10th percentile during the first 24 hours of life. Although there were differences during the first 24 hours of life in systolic $(34.4 \pm 5.3$ vs $35.2 \pm 3.4)$, diastolic $(17 \pm 3$ vs. $20 \pm 4$ torr $)$ and MBP ( $22 \pm 3$ vs. $24 \pm 3$ torr $)$ between the treatment and the control groups respectively, they were not statistically significant (Table 2).

Table 2 Comparison between Treated and Control Groups on Blood pressure

\begin{tabular}{llll}
\hline BP/Time & Treated $(\mathbf{N}=\mathbf{I}$ 7) & Control $(\mathbf{N}=\mid \mathbf{7})$ & P-value \\
\hline Systolic Day I & $34.4 \pm 5.3$ & $35.2 \pm 3.4$ & 0.55 \\
Diastolic Day I & $17.2 \pm 2.9$ & $17.9 \pm 2.7$ & 0.45 \\
Mean Day I & $22.9 \pm 3.2$ & $23.7 \pm 2.7$ & 0.41 \\
Systolic, Day 4 & $48.2 \pm 6.1$ & $48.6 \pm 7.2$ & 0.84 \\
Diastolic, Day 4 & $28.1 \pm 4.6$ & $30.2 \pm 7.3$ & 0.22 \\
Mean, Day 4 & $34.8 \pm 4.7$ & $36.3 \pm 6.9$ & 0.44 \\
Systolic, Day 7 & $50.3 \pm 5.6$ & $51.9 \pm 7.9$ & 0.47 \\
Diastolic, Day 7 & $30.1 \pm 4.5$ & $31.9 \pm 6.5$ & 0.34 \\
Mean, Day 7 & $36.8 \pm 4.4$ & $38.6 \pm 6.3$ & 0.35 \\
\hline
\end{tabular}

Values are expressed as Mean \pm SD

Lowest values for the day were entered.

All infants in the treatment group received one to three boluses of $20 \mathrm{ml} / \mathrm{kg}$ normal saline solution via the intravenous route over 30 minutes, four infants received Dopamine, and two of these 4 infants were also administered dobutamine infusion to a target MBP above the 10th percentile for their gestational age. Although the treatment group had higher incidences of PDA with left to right shunt, $(82.5 \%$ versus $76.5 \%$ ) the difference was not significant. All infants with PDA and left to right shunt were treated with indomethacin, and none was surgically ligated. There was significant increase in systolic, diastolic and MBP after PDA closure that continued to increase at day 4,7.

Repeated measurements of systolic, diastolic and mean blood pressure showed significant differences among subjects nested within each group (Table 3).

Table 3 Comparisons of average BP for the three time periods of the oneweek observation of BP

ANOVA Results for SBP, DBP and MBP

\begin{tabular}{lll}
\hline Source of variation & DF & p-value \\
\hline SBP & & \\
Subject nested within & 34 & $<0.0 I^{* *}$ \\
group & $\mathrm{I}$ & 0.72 \\
Group & 2 & $<0.0 I^{* *}$ \\
Time & & \\
DBP & & \\
Subject nested within & 34 & $<0.0 I^{* *}$ \\
group & $\mathrm{I}$ & 0.11 \\
Group & 2 & $<0.0 I^{* *}$ \\
Time & & \\
Mean BP & & \\
Subject nested within & 34 & $<0.0 I^{* *}$ \\
group & $\mathrm{I}$ & 0.07 \\
Group & 2 & $<0.0 I^{* *}$ \\
Time & & \\
\hline
\end{tabular}

**Significant at I\% level $(\mathrm{P}$-value<0.0I)
For SBP, DBP and Mean BP.

1. There were significant differences among subjects nested within group. This is not of interest as subjects are expected to be different for either group.

2. There was no significant difference between the Control and Treatment groups.

3. There were significant overall differences among the three time periods. Averaged over the control and treatment groups, the means for the three time periods and the significant 2-time comparisons (using Bonferroni adjustment of significance level) are as follows.

\begin{tabular}{lr}
\hline \multicolumn{3}{l}{ Bonferroni's multiple comparisons } \\
\hline SBP & First 3 Days (35.11) \\
DBP $\quad$ First 3 Days (17.47) \\
Mean BP First 3 Days (23.42)
\end{tabular}

Above, a line connecting two time periods indicate no significant difference. Two time periods are significantly different if there is no line connecting them.

For SBP, the significant time comparisons are those between Time 1 (First 3 days) and Time 2 (Day 4 ) and between Time 1 (First 3 days) and Time 3 (Day 7). There is no significant difference in average SBP between Day 4 and Day 7.

For DBP, the significant time comparisons are those between Time 1 (First 3 days) and Time 2 (Day 4) and between Time 1 (First 3 days) and Time 3 (Day 7). There is no significant difference in average DBP between Day 4 and Day 7.

For Mean BP, the significant time comparisons are those between Time 1 (First 3 days) and Time 2 (Day 4) and between Time 1 (First 3 days) and Time 3 (Day 7). There is no significant difference in average Mean BP between Day 4 and Day 7.

There were no differences between the control and the treatment group at day 4 and day 7 respectively (Figures 1-3).

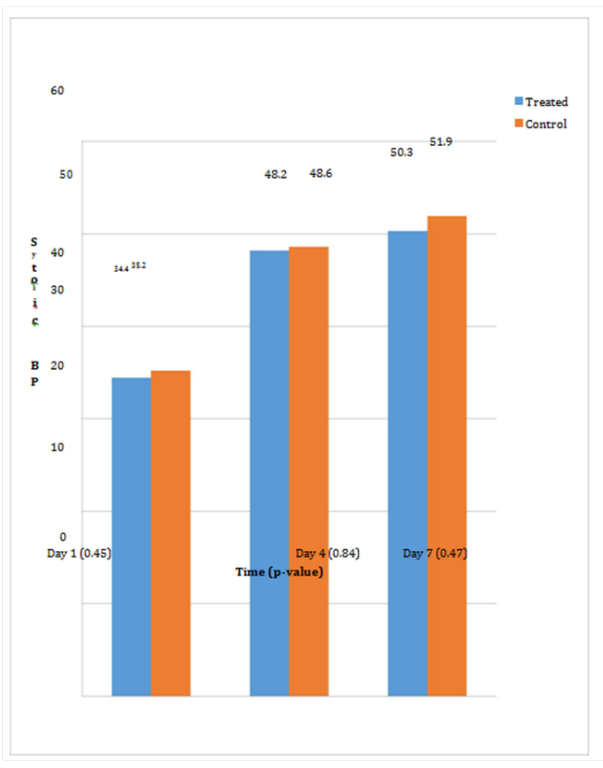

Figure I Systolic Blood Pressure Comparison between Control and Treated Newborns by Time 


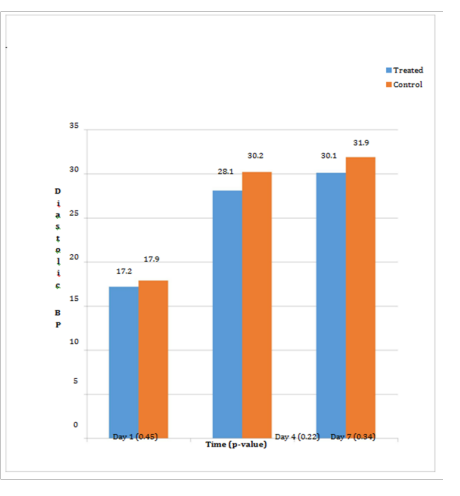

Figure 2 Diastolic Blood Pressure Comparison between Control and Treated Newborns by Time

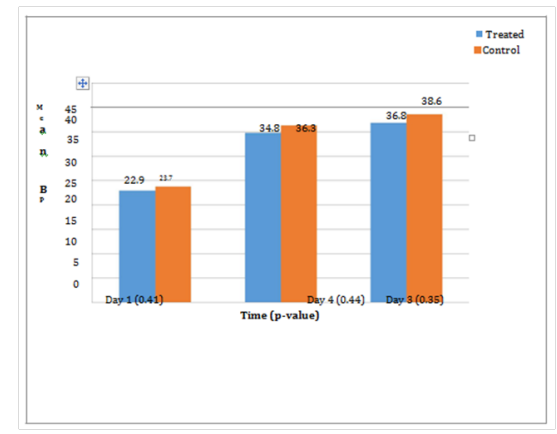

Figure 3 Mean Blood Pressure Comparison between Control and Treated Newborns by Time

\section{Secondary outcomes}

The incidence of IVH, shown in table 4, was statically significant in the treatment group: nine $(52.9 \%)$ versus three $(17.6 \%)$ in the control group respectively $(P>0.03)$. There was a high incidence of BPD in the treatment and control groups, $13(81 \%)$ vs. 12

(71\%) respectively $(P>0.69)$. ROP was higher in both groups, but was not statistically significant. Three infants in the treatment group died at day 14, 19, and 32 respectively due to NEC complications while two infants in the control group died at days 9 and 12 of NEC. This difference in mortality was not statically significant (Table 4).

Table 4 Difference in mortality was not statically significant

\begin{tabular}{llll}
\hline & Treatment & Control & P Value \\
\hline IVH & $9(52.9 \%)$ & $3(17.6 \%)$ & $0.03 \dagger$ \\
BPD & $13(81 \%)$ & $12(70.6 \%)$ & 0.69 \\
ROP & $12(70.6 \%)$ & $10(58.80 \%)$ & 0.72 \\
PDA & $14(82.4 \%)$ & $12(76.5 \%)$ & 0.69 \\
NEC & $3(17.6 \%)$ & $2(11.8 \%)$ & 0.99 \\
Mortality & $3(17.6 \%)$ & $2(11.8 \%)$ & $>0.99$ \\
\hline
\end{tabular}

$\dagger$ Significant at $5 \%$ level $(0.01<\mathrm{P}$-value $<0.05)$

\section{Discussion}

Blood pressure is the product of stroke volume and systemic vascular resistance; however, the immature myocardium of ELBW infants has incomplete sympathetic innervations and less contractile tissue mass, leading to less reserve to adapt to the volume overload due to left to right shunting via a PDA with a resultant decrease in systolic blood pressure. A PDA will also cause a decrease in the diastolic blood pressure by reducing the systemic vascular resistance. ${ }^{1,7}$

\section{Discussion on measured limitations}

In this prospective, randomized study, we compared the strategy of targeting MBP above the 10th percentile for gestational age in stable ELBW infants during the first day of life to observation alone while monitoring systolic, diastolic and MBP during the first week of life. We further examined the impact of PDA closure on the ABV on day 4, and 7 of life respectively. Infants in the treatment group were managed according to the inherited use of volume support and vasopressors by the NICU attending versus observation in the control group. We found associations between PDA with mild to moderate left to right shunt, and decreases in systolic, diastolic, and mean blood pressure. Treatment of the PDA led to increase in systolic, diastolic and MBP, the increase in $\mathrm{ABV}$ continued on day 4 and 7 of life. The increase in the ABVs was statistically significant within each group but not between the control and treatment groups. Our results are consistent with a recent study of Miall-Allen et al., ${ }^{14}$ that ABV decreased in the first 4 hours of life then increased and that rise of $A B V$ was similar for untreated infants and infants given any anti-hypotensive therapy (fluid bolus and/or vasopressor). However in Beau's study they did not evaluate the effects/association of PDA with left to right shunt on $\mathrm{ABV}$ in those ELBW who had ABV at 24 hours less than ABV at 4 hour of life.

Ratner et al. ${ }^{5}$ and Evan et al. ${ }^{6}$ have shown that a hemodynamically significant PDA is associated with a reduction in both systolic and diastolic blood pressure with a resultant decrease of the MBP. Our study found an association between low MBP and PDA, with the systolic, diastolic and the MBPs all increasing after the closure of the PDA. Systolic, diastolic and the MBP continued to increase over the first week of life. The increase in ABVs were similar between the treatment and the control groups but was statistically significantly different within the groups after PDA closure. The increased incidence of IVH in the treatment group may be explained by rapid volume expansion in an attempt to increase MBP which is in agreement with the study done by Goldberg et al. ${ }^{15,16}$ Although, we found no difference in BPD, ROP, NEC, and mortality between the two groups the numbers are inadequate.

Our data suggest that in stable premature infants low blood pressure observed initially resolve with time without treatment. Furthermore, we found an association between PDA and Low Mean Blood Pressure in stable ELBW infants. PDA Management may be considered before attempting to treat the low MBP with blood volume expanders or vasopressors.

This pilot study support a large randomized control study of observation showed that initial low blood pressures variables increase with time, without intervention.

\section{Acknowledgements}

None.

\section{Conflict of interest}

The authors have no conflict of interest to disclose. 


\section{References}

1. Seri I, Evans J. Controversies in the diagnosis and management of hypotension in the newborn infant. Curr Opin Pediatr. 2001;13(2):116123.

2. Cunningham S, Symon AG, Elton RA, et al. Intra-arterial blood pressure reference ranges, death and morbidity in very low birth weight infants during the first seven days of life. Early Hum Dev. 1999;56(2-3):151-165.

3. Batton B, Batton D, Riggs T. Blood pressure in the first 7 days in premature infants born at postmenstrual age 23 to 25 weeks. Am J Perinatol. 2007;24(2):107-115.

4. Levene M, Chiswick M, Field D, et al. Development of audit measures and guidelines for good practice in the management of neonatal respiratory distress syndrome. Arch Dis Child. 1992;67:1221-1227.

5. Ratner I, Perelmuter B, Toews W, et al. Association of low systolic and diastolic blood pressure with significant patent ductus arteriosus in very low birth infants. Crit Care Med. 1985;13(6):497-500.

6. Evans N, Iyer P. Assessment of ductus arteriosus shunt in preterm infants supported by mechanical ventilation: effects of interarterial shunting. $J$ Pediatar. 1994;125(5 Pt 1):778-785.

7. Versmold HT, Kitterman JA, Phibbs RH, et al. Aortic blood pressure during the first $12 \mathrm{~h}$ of life in infants with birth weight 610-4220 grams. Pediatrics. 1981;67(5):607-613.

8. Spinazzola RM, Harper RG, de Soler M, et al. Blood pressure values in 500- to 750-gram birthweight infants in the first week of life. J Perinatol. $1991 ; 11(2): 147$.
9. Hegyi T, Anwar M, Carbone MT, et al. Blood pressure ranges in premature infants: II. The first week of life. Pediatrics. 1996;97(3):336-342.

10. Nuntnarumit P, Yang W, Bada-Ellzey HS. Blood pressure measurements in the newborn. Clin Perinatol. 1999;26:981-996.

11. Bada HS, Korones SB, Perry EH, et al. Mean arterial blood pressure changes in premature infants and those at risk for intravascular hemorrhages. $J$ Pediatar. 1990;117:607-614.

12. Meek JH, Tyszczuk L, Elwell CE, et al. Low cerebral blood flow is a risk factor for severe intraventricular hemorrhage. Arch Dis Child Fetal Neonatal Ed. 1999;81(1):F15-F18.

13. Bell MJ, Ternberg JL, Feigin RD, et al. Neonatal necrotizing enterocolitis: therapeutic decisions based upon clinical staging. Ann Surg. 1978;187(1):1-

14. Miall-Allen VM, Whitelaw AG. Response to dopamine and dobutamine in preterm infant less than 30 weeks' gestation. Crit Care Med. 1989;17(11):1166-1169

15. Goldberg RN, Chung D, Goldman SL, et al. The association of rapid volume expansion and intraventricular hemorrhage in the preterm infant. $J$ Pediatr. 1980;96(6):1060-1063.

16. Watkins AM, West CR, Cooke RW. Blood pressure and cerebral hemorrhage and ischemia in very low birth-weight infants. Early Hum Dev 1989;19(2):103-110. 